\title{
Radiation characteristics of helical tomotherapy
}

\author{
Robert Jeraj ${ }^{\mathrm{a})}$ \\ Department of Medical Physics, University of Wisconsin-Madison, 1530 MSC, 1300 University Avenue, \\ Madison, Wisconsin 53706, TomoTherapy Inc., 1240 Deming Way, Madison, Wisconsin 53717, \\ and Jozef Stefan Institute, Jamova 39, SI-1000 Ljubljana, Slovenia
}

Thomas R. Mackie, John Balog, and Gustavo Olivera

Department of Medical Physics, University of Wisconsin-Madison, 1530 MSC, 1300 University Avenue, Madison, Wisconsin 53706 and TomoTherapy Inc., 1240 Deming Way, Madison, Wisconsin 53717

Dave Pearson, Jeff Kapatoes, Ken Ruchala, and Paul Reckwerdt

TomoTherapy Inc., 1240 Deming Way, Madison, Wisconsin 53717

(Received 15 July 2003; revised 13 November 2003; accepted for publication 14 November 2003; published 27 January 2004)

Helical tomotherapy is a dedicated intensity modulated radiation therapy (IMRT) system with on-board imaging capability (MVCT) and therefore differs from conventional treatment units. Different design goals resulted in some distinctive radiation field characteristics. The most significant differences in the design are the lack of flattening filter, increased shielding of the collimators, treatment and imaging operation modes and narrow fan beam delivery. Radiation characteristics of the helical tomotherapy system, sensitivity studies of various incident electron beam parameters and radiation safety analyses are presented here. It was determined that the photon beam energy spectrum of helical tomotherapy is similar to that of more conventional radiation treatment units. The two operational modes of the system result in different nominal energies of the incident electron beam with approximately $6 \mathrm{MeV}$ and $3.5 \mathrm{MeV}$ in the treatment and imaging modes, respectively. The off-axis mean energy dependence is much lower than in conventional radiotherapy units with less than $5 \%$ variation across the field, which is the consequence of the absent flattening filter. For the same reason the transverse profile exhibits the characteristic conical shape resulting in a 2-fold increase of the beam intensity in the center. The radiation leakage outside the field was found to be negligible at less than $0.05 \%$ because of the increased shielding of the collimators. At this level the in-field scattering is a dominant source of the radiation outside the field and thus a narrow field treatment does not result in the increased leakage. The sensitivity studies showed increased sensitivity on the incident electron position because of the narrow fan beam delivery and high sensitivity on the incident electron energy, as common to other treatment systems. All in all, it was determined that helical tomotherapy is a system with some unique radiation characteristics, which have been to a large extent optimized for intensity modulated delivery. (c) 2004 American Association of Physicists in Medicine. [DOI: 10.1118/1.1639148]

Key words: Monte Carlo simulations, helical tomotherapy, beam characteristics

\section{INTRODUCTION}

While some of the physical and dosimetric characteristics of photon and electron radiation therapy units can be measured, the most complete description of the radiation field is provided through Monte Carlo simulations. Therefore, it is not surprising that much effort has been put in detailed, accurate and versatile simulations of almost all currently existing radiotherapy units in the last two decades. ${ }^{1-30}$ Monte Carlo simulations were initially of a more qualitative nature because of long calculation times and difficulties in specifying complex geometry of a linear accelerator head. Particularly with the efforts of the OMEGA project and introduction of the BEAM code ${ }^{31}$ the simulations became much more quantitative and widespread. Besides of the BEAM code, based on the EGS Monte Carlo transport code ${ }^{32,33}$ several other codes, like MCNP,${ }^{34}$ PENELOPE,${ }^{35}$ PEREGrine, ${ }^{11}$ have been used as well.
Most of the photon radiation therapy units have a very similar structure, composed of a high atomic number target, where the incoming electron beam from the linac creates bremsstrahlung radiation, primary collimator, where the beam is shaped into the maximum field size and shielded outside, flattening filter, where the radiation field is flattened, monitor chamber, where the output is monitored; and the secondary collimator, where the beam is shaped into the final shape. Optionally, additional beam modulators like wedges, compensators or multileaf collimators are used to modulate the beam intensity of an open field.

In this work a recently developed and in some aspects nonstandard radiotherapy system-helical tomotherapy ${ }^{36}$ is investigated. Helical tomotherapy is the first treatment unit dedicated to intensity modulated radiotherapy (IMRT) and a fully integrated image guided radiotherapy system with the on-board mega-voltage CT (MVCT) capability. It was devel- 
oped and constructed at the University of WisconsinMadison and TomoTherapy Inc., Madison, WI. The first prototype investigated in this study was installed at University of Wisconsin Hospital and Clinics in Madison, WI, USA. It should be mentioned that the prototype (Hi-ART I) and subsequent commercial units (Hi-ART II) do not differ significantly in their radiation characteristics; therefore, the results of this work should be directly applicable to current commercial units. All the potential differences between the two systems are explicitly mentioned.

The basic radiation source of the helical tomotherapy system is a linear accelerator mounted on a rotating gantry similarly to a CT scanner. The radiation is delivered to a patient in a helical way, obtained by concurrent gantry rotation and couch/patient travel. The treatment field is modulated with a 64-leaf binary multileaf collimator. Contrary to other systems the source to axis distance (SAD) is $85 \mathrm{~cm}$ instead of the usual $100 \mathrm{~cm}$. The radiation field has a fan shape with the maximum transverse dimension of $40 \mathrm{~cm}$ and variable lateral dimension (i.e., slice thickness) of up to $5 \mathrm{~cm}$, defined by the jaw (secondary collimator) opening. Because the helical tomotherapy system is a dedicated IMRT system it differs in several ways from other radiotherapy systems. The most important difference are the absence of a flattening filter, a thin target, an electron stopper, a beam hardener and a compact primary collimator, which cause the radiation field to be significantly different from that of other treatment units.

Because of the mentioned differences relative to a standard radiotherapy machine, the main focus of this paper is on the description of radiation characteristics of the helical tomotherapy unit, especially in parts where it differs from standard accelerators.

\section{MATERIALS AND METHODS}

The Monte Carlo model of the helical tomotherapy treatment unit was based on detailed mechanical specifications of the linac head components and the materials used in manufacturing. For this reason, the number off unknown parameters in the model was minimal and the accuracy of the model uncompromised. In addition to the treatment head, a binary multileaf collimator has been modeled, also including all the details. It should be emphasized that the model was constructed with the accuracy that surpasses the accuracy of other treatment unit models. The reason is that all the manufacturer's data was fully disclosed. The Monte Carlo model of the helical tomotherapy treatment unit head is shown in Fig. 1.

The Monte Carlo code used in our studies was MCNP4c $3^{34}$ with its default cross section libraries. The MCNP code was chosen because of the flexible combinatorial geometry capabilities required for detailed modeling of the system. However, no significant differences in the simulation results would be expected if an alternative Monte Carlo code, like EGS4 BEAM would be used. ${ }^{26}$ In the simulations, the only free parameter that had to be tuned were characteristics of the incident electron beam on the target (spatial, angular and energy distribution). The electron beam incident on the target

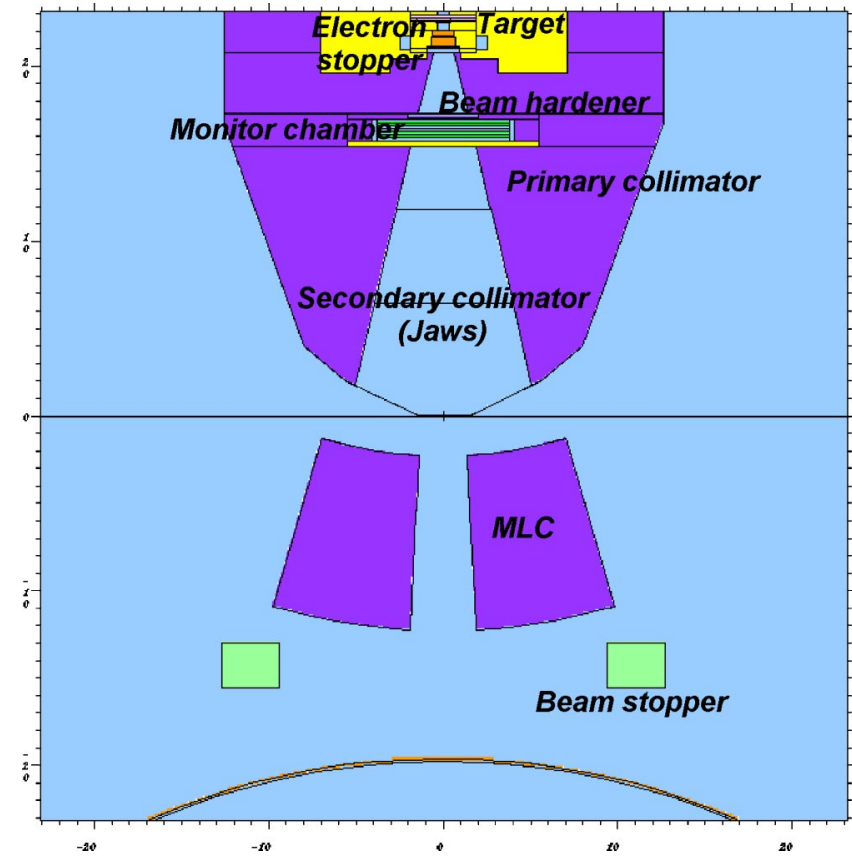

FIG. 1. Model of the helical tomotherapy unit as used in the Monte Carlo simulations. No geometrical or material simplifications were introduced in order to avoid systematic errors in the results. Different structures within the head are marked. The phase space scoring plane below the primary collimator is shown.

was modeled to be Gaussian in energy and spatial distribution with the normal incident direction.

Three phase space scoring planes were used in the complete Monte Carlo model of the helical tomotherapy unit. The first one was placed between the treatment head and multileaf collimator, the second one after the multileaf collimator (before the patient) and the third one after the patient (before the detector). Since in this work only radiation characteristics of the radiation treatment unit were investigated, only the upper part of the model was applicable (see Fig. 1). The following variance reduction methods were used in order to increase the simulation speed: geometry celldependent energy cut off, geometry importance splitting/ Russian roulette and bremsstrahlung splitting. At least $10^{9}$ source particles were used for phase space calculation, which was typically 10-times recycled for dose calculations. All the simulations were performed at the open field geometry conditions, which corresponds to a $5 \times 40 \mathrm{~cm}^{2}$ field at an $85 \mathrm{~cm}$ surface to source distance, except where stated otherwise. Scoring regions for depth dose calculations were $10 \times 10$ $\times 5 \mathrm{~mm}^{3}$. The average spectra were scored in the central 4 $\times 40 \mathrm{~cm}^{2}$ area, except for the off-axis dependence where the scoring regions were $4 \times 5 \mathrm{~cm}^{2}$. Resolution in the dose profile calculations was $5 \mathrm{~mm}$ for the transverse (in-slice) profile and $1 \mathrm{~mm}$ for the lateral (across-slice) profile. Sensitivity studies on the incident electron beam position and direction were performed with $0.5 \mathrm{~mm}$ resolution. Statistical errors in Monte Carlo simulations were kept below $1 \%$ for most of the simulations. Because of the low statistical error, no error bars are shown in the results.

All the measurements used in this study were also per- 
formed at the open field geometry conditions. Depth dose distributions were measured with an Exradin A12 farmer chamber (Standard Imaging, Middleton, WI) in a motor controlled TG51 calibration system (Standard Imaging, Middleton, WI). Kodak EDR2 Ready-Pack film (Eastman Kodak Company, Rochester, NY) was used for profile measurements in custom made Solid Water slabs (Gammex Inc., Middleton, WI). A Howtek MultiRAD 450 film scanner (Howtek, Hudson, NH) was used for film scanning. Measurement errors were estimated to be below $1 \%$; therefore, they are not shown in the results.

The Monte Carlo model was commissioned using the measured dose distributions using a standard iterative commissioning procedure. During this procedure, the incident electron beam was characterized. In all subsequent studies these parameters were used. For comparison to other systems, two Varian Clinac treatment units (600C/D and 2100C) were simulated. While the geometric models of the Varian units were accurate (according to the manufacturers standard Monte Carlo geometry specifications), the incident electron beam parameters were assumed to be the same in all units (incident energy $6 \pm 0.5 \mathrm{MeV}$, uniform radial distribution with the diameter of $2 \mathrm{~mm}$ ).

\section{RESULTS}

\section{A. Monte Carlo model commissioning}

The first step in the Monte Carlo modeling was commissioning of the model to match the dose measurements. The depth dose curve and transverse dose profiles were used as the main dosimetric quantities during Monte Carlo model commissioning. The best fit with the experimental values was obtained with incident electron energy of $5.7 \mathrm{MeV}$. The energy (Gaussian) spread was found to be rather unimportant. The standard deviation was kept at approximately 5\% of the nominal energy, or $0.3 \mathrm{MeV}$ in our case. The radial dependence of the incident electron beam was also modeled to be Gaussian with the FWHM of $1.4 \mathrm{~mm}$. The comparison between the commissioned beam and measurements is shown in Fig. 2.

In addition to tuning the incident electron beam parameters, it is possible to calculate the Monte Carlo model calibration factor $N_{\mathrm{MC}}$, or the number of incident electrons on the target. The comparison was performed for the 10 $\times 5 \mathrm{~cm}^{2}$ calibration field, $85 \mathrm{~cm} \mathrm{SSD} \mathrm{and} \mathrm{point} \mathrm{of}$ measurement/calculation at $10 \mathrm{~cm}$ depth. The average dose rate at $D_{\max }, \dot{D}$ is about $10 \mathrm{~Gy} / \mathrm{min}$. The Monte Carlo calibration factor $N_{\mathrm{MC}}$, can be calculated from the ratio of the measured dose per pulse $D_{\text {meas }} /$ pulse (or the ratio of the measured dose rate and pulse frequency $\dot{D} / \nu$ ) and Monte Carlo calculated dose per source particle (incident electron) $D_{\mathrm{MC}} /$ s.p.,

$$
N_{\mathrm{MC}}=\frac{D_{\text {meas }} / \text { pulse }}{D_{\mathrm{MC}} / \text { s.p. }}=\frac{\dot{D} / \nu}{D_{\mathrm{MC}} / \mathrm{s} \cdot \mathrm{p}} .
$$

In the case of helical tomotherapy this results in 3.2 $\times 10^{12} e^{-} /$pulse. However, this number should be seen only
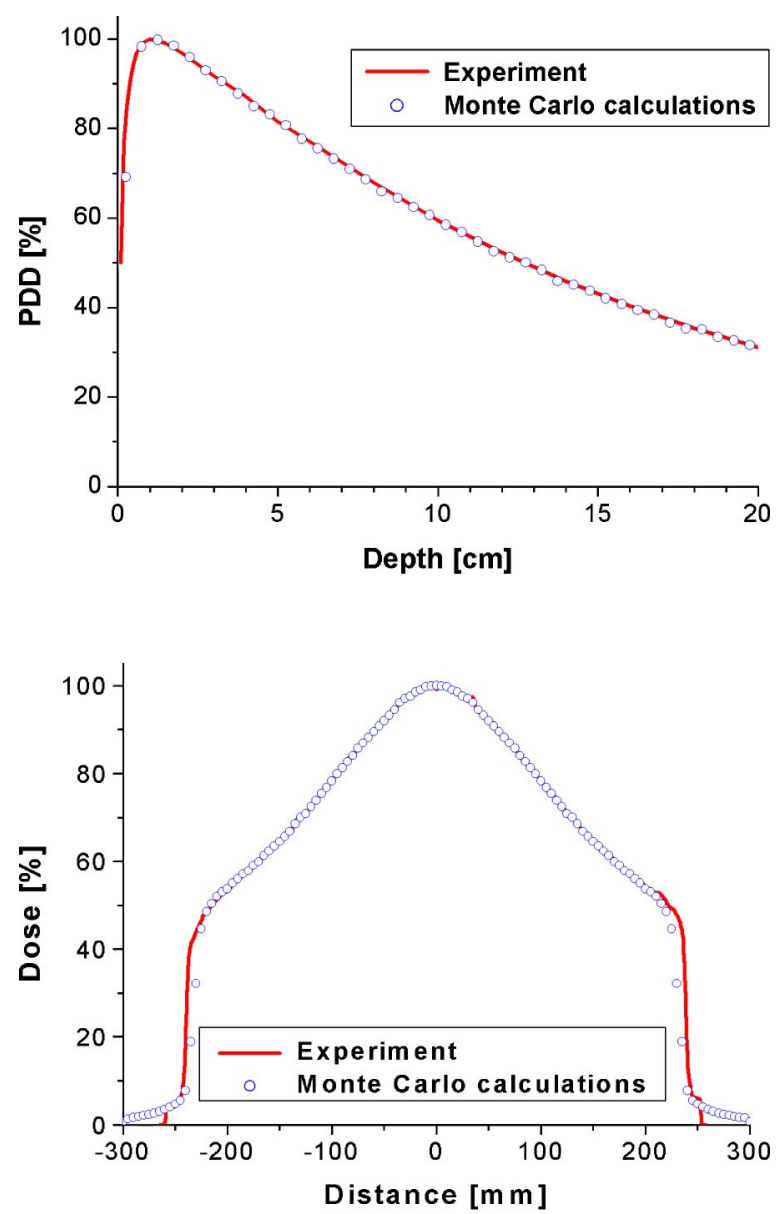

FIG. 2. A comparison of Monte Carlo calculations and measurements of the central axis percent depth dose (PDD) distributions (top) and transverse (in-slice) profiles (bottom). The ionization chamber was used for depth dose measurements and film for the profiles. The statistical error of Monte Carlo simulations was below $1 \%$ in both cases.

as an estimate since the linac electron fluence output can be adjusted with the variation of the order of $10 \%$. The incident electron beam parameters and calibration factor determined through Monte Carlo model commissioning were used through our studies, except where stated otherwise.

\section{B. Photon energy spectrum}

The single most important characteristic of any radiation field is its energy spectrum. Even though the helical tomotherapy system is significantly different from other radiotherapy units, its spectrum is rather comparable to Varian Clinac spectra for the beams of the same nominal energy as seen in Fig. 3.

It is expected that the energy spectra should not be too different, since most of the spectrum shape is dominated by the characteristic bremsstrahlung spectrum. Only the spectrum details are dependent on the geometry details, especially in the lower energy part of the spectrum.

Another important characteristic of the photon spectrum is its angular dependence. While it is known that in conventional treatment linacs the off-axis beam softening (or equivalently central-axis beam hardening) is substantial and 


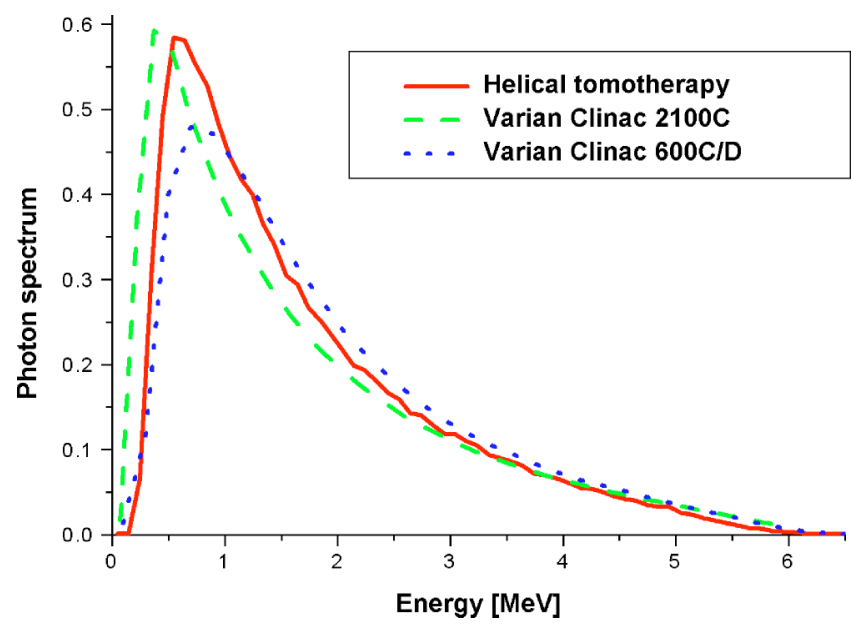

FIG. 3. Photon energy spectrum of helical tomotherapy, compared to two different Varian Clinac units, 2100C and 600C/D. All of the systems were set to have the same incident electron beam (incident energy $6 \pm 0.5 \mathrm{MeV}, 2$ $\mathrm{mm}$ in diameter). The spectra are normalized to better show spectral characteristics.

can be of the order of $30 \%-40 \%,{ }^{15}$ there is very little offaxis spectrum changes in the helical tomotherapy beam (Fig. 4). The mean energy of the photon beam, which is $1.49 \mathrm{MeV}$ in the center decreases by only about $5 \%$ to $1.43 \mathrm{MeV}$ for the most distant part of the fan beam (about $20 \mathrm{~cm}$ off-axis). The reason for this small difference is the absence of the flattening filter, which contributes most of the off-axis spectral dependence. It should be emphasized that the almost negligible off-axis spectral dependence is very favorable for commissioning of the convolution/superposition dose calculation algorithm used in the treatment planning system.

Because the helical tomotherapy system plays a dual role-treatment and imaging, there are also two different modes of operation-treatment mode and MVCT imaging mode. In the MVCT imaging mode the energy of the incident electron beam is reduced in order to provide the beam with better imaging characteristics (better contrast per given dose). The spectra of both modes are shown in Fig. 5. The best energy match with the experimental data was approximately $3.5 \mathrm{MeV}$ for the MVCT imaging mode. This leads in the average photon beam energy of about $1.5 \mathrm{MeV}$ for the treatment mode and $1.0 \mathrm{MeV}$ for the imaging mode.

\section{Photon fluence shape}

One of the most important differences of the helical tomotherapy system compared to other radiation therapy systems is that it does not have a flattening filter, which would make the dose at depth more uniform. This results in the characteristic conical-shaped transverse (in-slice) fluence profile shown in Fig. 6.

The main reason for allowing the nonuniform profile is that helical tomotherapy is a dedicated IMRT system, without a need for the flat dose profile. If still desired, the multileaf collimator can be used to modulate the treatment field to produce a flat dose distribution. On the other hand, as seen from Fig. 6, the absence of the flattening filter increases the
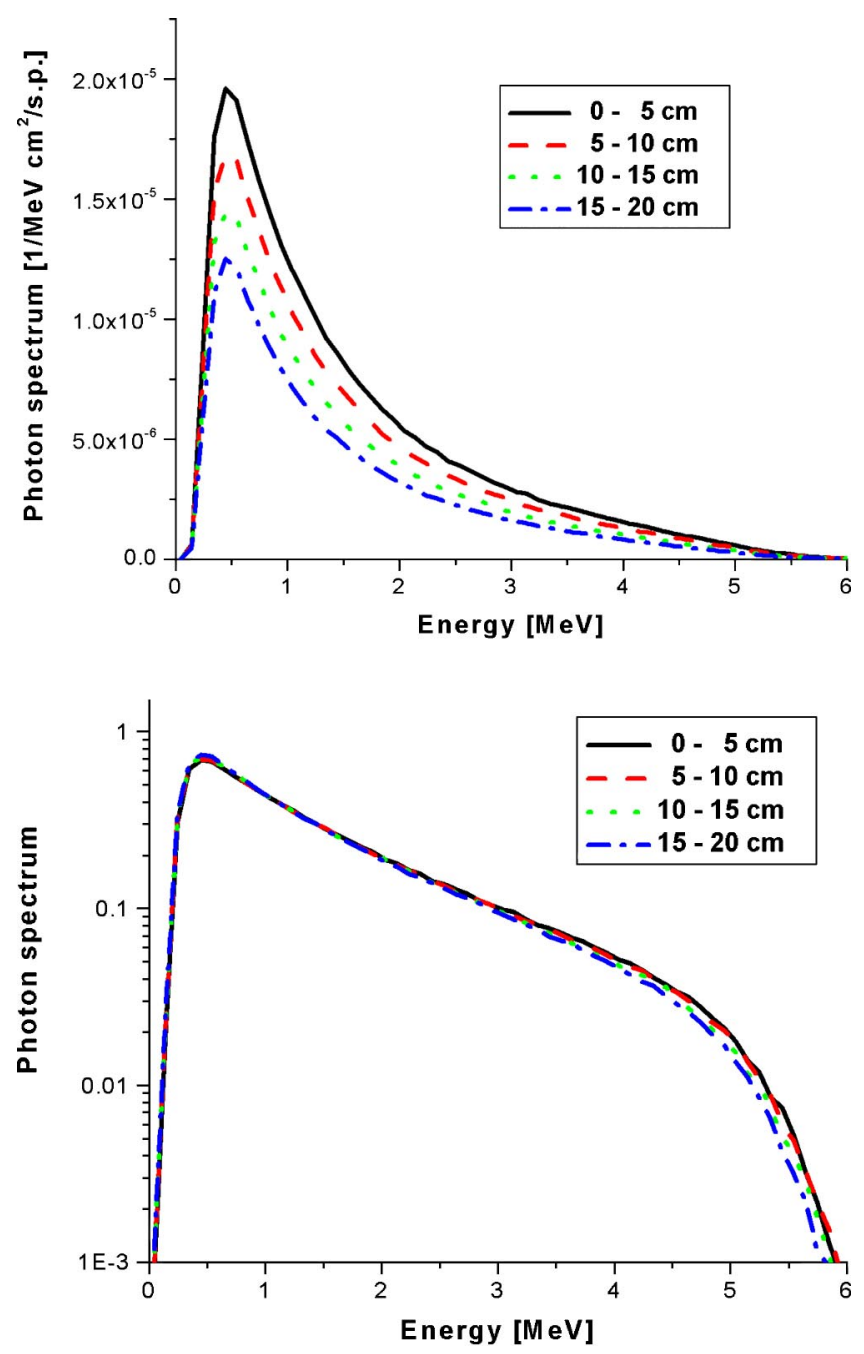

FIG. 4. Absolute (top) and normalized (bottom, log-lin scale) off-axis spectral dependence of the helical tomotherapy treatment beam. The incident electron energy was $5.7 \mathrm{MeV}$ and the radial spread was $1.4 \mathrm{~mm}$ (FWHM). Note very little off-axis spectral change (less than $5 \%$ with the average energy ranging from $1.43 \mathrm{MeV}$ at the edge of the field to $1.49 \mathrm{MeV}$ in the center), which is very different compared to other radiotherapy systems. The reason for small dependence is the absence of the flattening filter.

dose output in the center of the field by approximately twofold compared to the edge of the field. This leads to an increased average dose rate and consequently reduced treatment times for patients.

Since the maximum slice thickness of the helical tomotherapy system is approximately $5 \mathrm{~cm}$, the conical fluence shape, as observed in the transverse profile, is hardly noticeable in the lateral (across-slice) fluence profile, as shown in Fig. 7. On the other hand, because the helical tomotherapy delivery is a narrow slice delivery, when fields smaller than approximately $2 \mathrm{~cm}$ are used, the intensity is reduced because of the obscured virtual source. The blocking of the virtual source becomes particularly noticeable, when the incident electron beam is misplaced from the center.

The sensitivity of the lateral beam profile to the incident electron beam position (Fig. 8) is higher for shifts than it is for tilts from central and normal incidence. Another interest- 


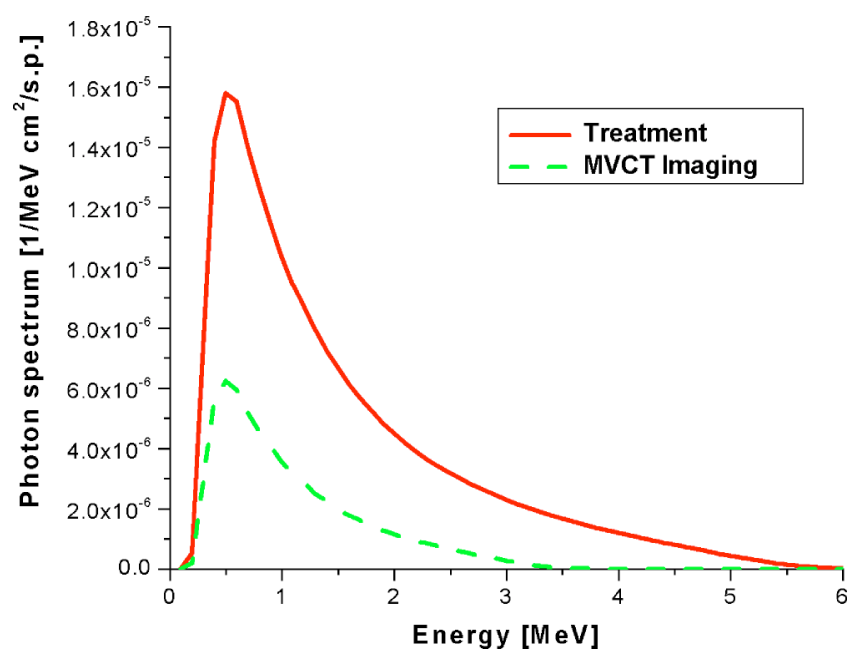

FIG. 5. The average photon beam spectra of helical tomotherapy for two operational modes: treatment mode and MVCT imaging mode. While in the treatment mode the incident electron energy is approximately $5.7 \mathrm{MeV}$, in the MVCT imaging mode it is reduced to about $3.5 \mathrm{MeV}$, corresponding to the average energies of $1.5 \mathrm{MeV}$ and $1.0 \mathrm{MeV}$, respectively.

ing detail is that even for the normal incidence the lateral profile is shifted, which appears from the tongue and groove of the jaws (primary collimator). It should be mentioned that based on this study, the jaws were redesigned, so that the sensitivity to the mis-position was reduced. In addition, tongue and groove was removed from the width-defining jaws, so that all commercial tomotherapy systems, except of the investigated helical tomotherapy research prototype, do not exhibit any asymmetry in the lateral fluence profile. The sensitivity of the transverse beam profile is much smaller because of the larger field size in the transverse direction and can be neglected.

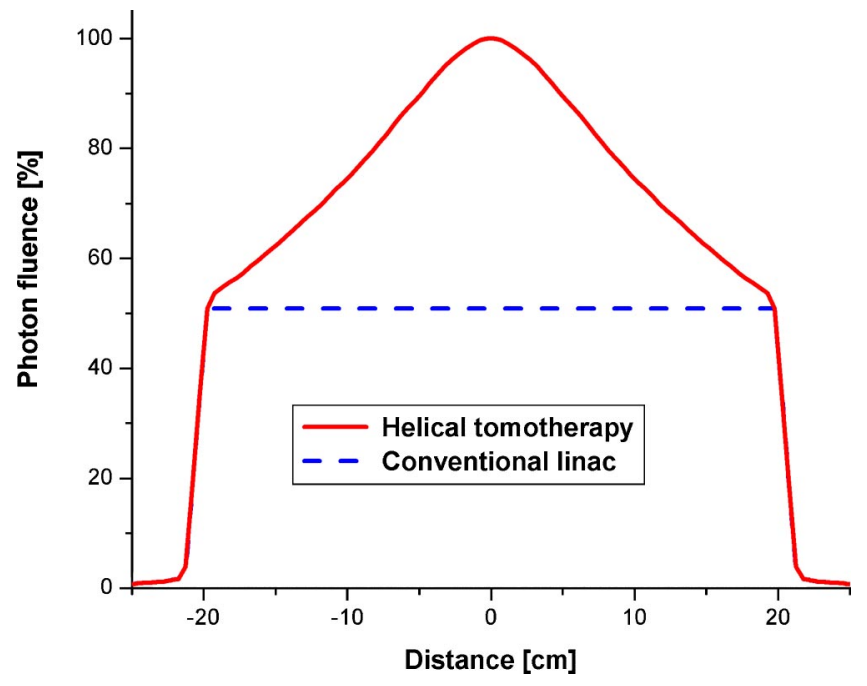

FIG. 6. The transverse (in-slice) photon fluence profile of the helical tomotherapy treatment beam. The characteristic conical shape of the profile is notable, which, on the contrary to the conventional treatment units (dashed), results in almost doubled photon fluence in the center of the beam.

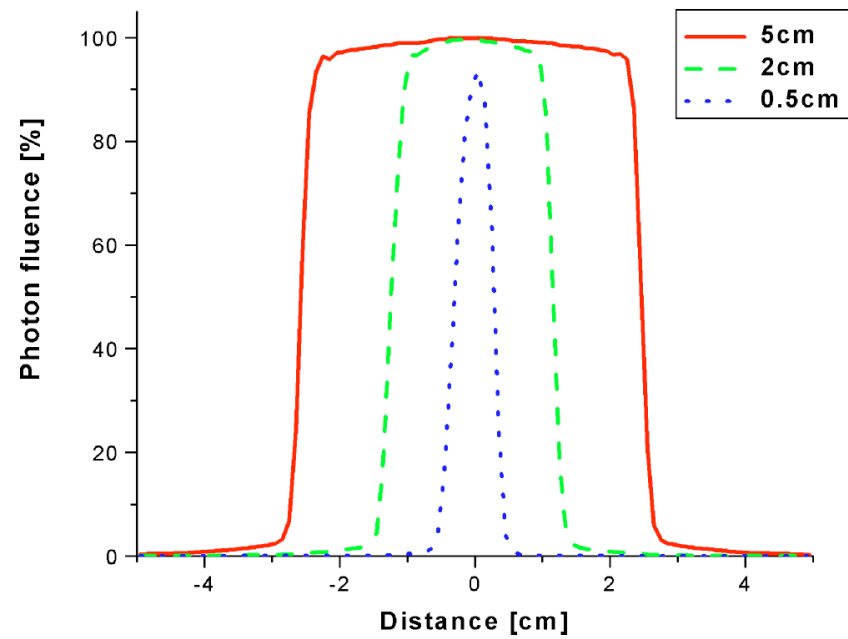

FIG. 7. Lateral (across-slice) photon fluence profile of the helical tomotherapy treatment beam for different slice thicknesses. While the pronounced conical shape in the transverse photon fluence profile is noticeable, the lateral photon fluence profile is nearly uniform.

\section{Leakage and scatter}

A very common concern in all forms of IMRT is the leakage radiation outside the radiation field. During delivery of
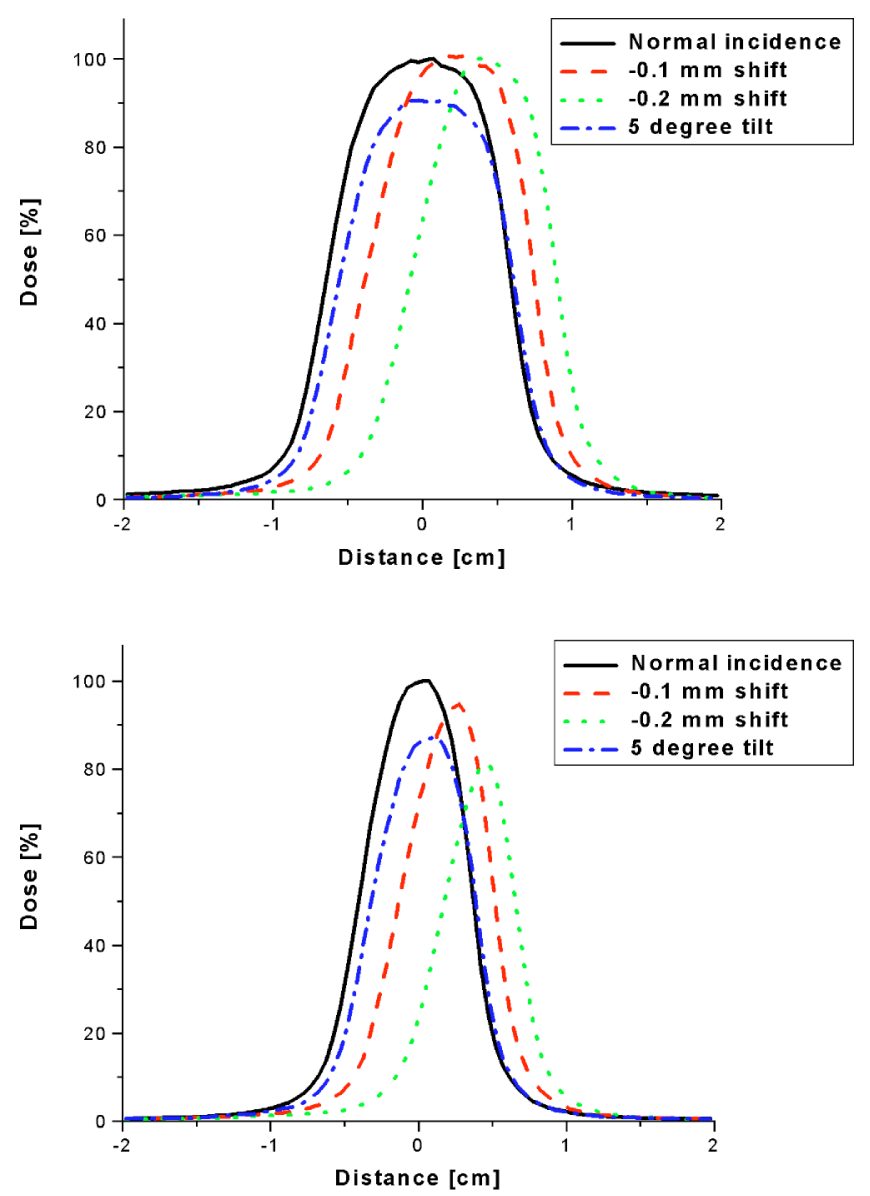

FIG. 8. Sensitivity of the lateral dose profile to the incident electron beam position. The sensitivity study was done for $1 \mathrm{~cm}$ (top) and $6 \mathrm{~mm}$ (bottom) slice thicknesses to increase the sensitivity. Note much stronger sensitivity to the shift than the tilt from normal incidence. 

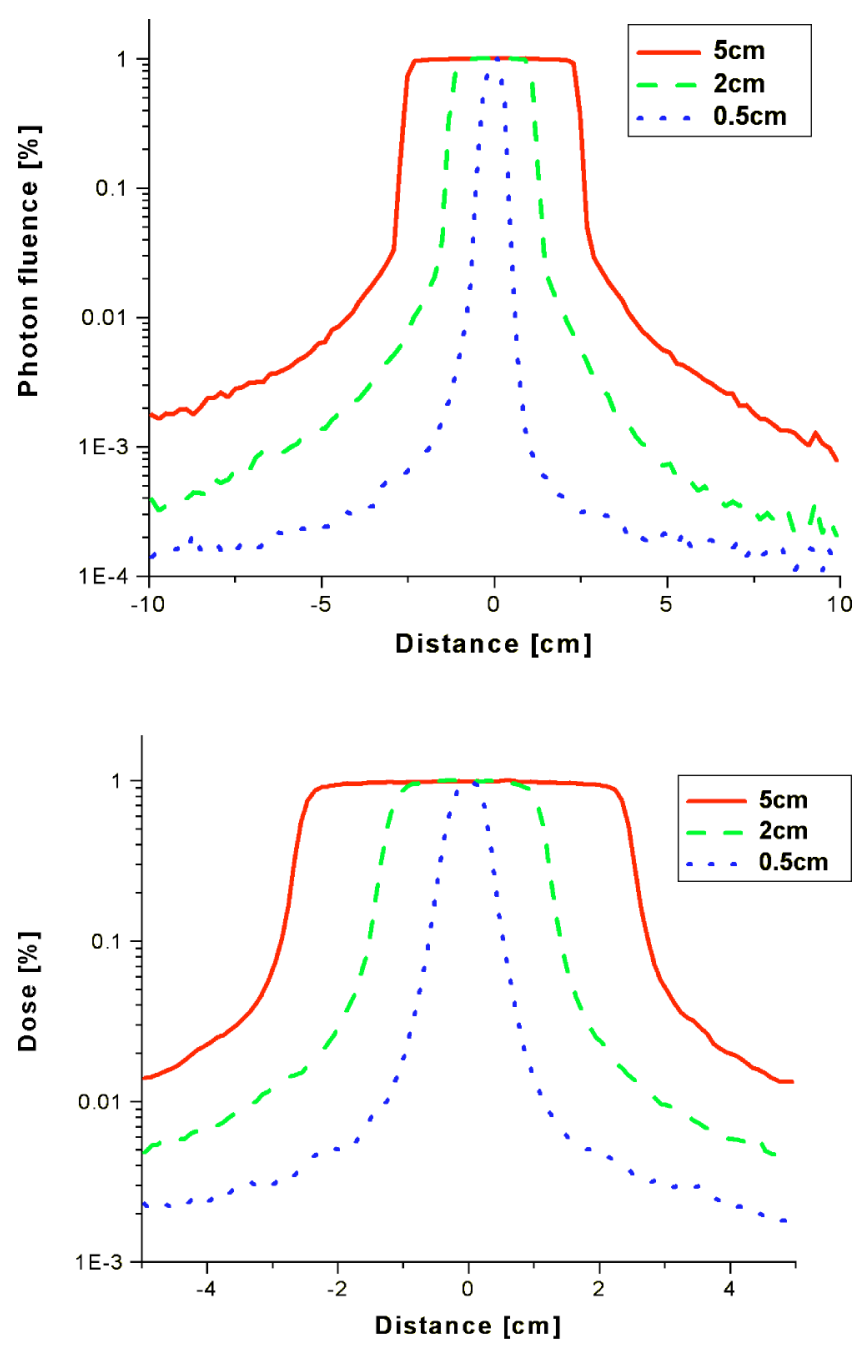

FIG. 9. Photon fluence (top) and dose profiles at $85 \mathrm{~cm}$ with $1.5 \mathrm{~cm}$ buildup (bottom) indicating fields inside and outside the radiation field. Slight asymmetry in the profiles because of the tongue and groove of the jaws is characteristic only for the prototype and does not exist in commercial units.

IMRT fields the small leakage radiation is summed up, and this could lead into significant dose levels outside the treatment region. ${ }^{37}$ The radiation in and outside the field for various slice thicknesses is shown in Fig. 9.

It can be seen that the leakage radiation is very low, primarily because of the increased shielding (up to $20 \mathrm{~cm}$ of tungsten) of the collimators, so that the main source of the radiation outside the field is the in-field scattered radiation. Since the scatter is smaller for narrower slices, a narrow slice delievery would be preferable; however, in practice it would result in approximately the same amount of scatter because of the multiple rotations needed to deliver a field of a certain length.

\section{E. Photon output fluence rate}

Important difference between helical (and serial) tomotherapy systems and other systems is that the delivered beam is of a narrow fan shape. Provided that the linac output fluence rates are the same, one could expect longer delivery times in case of tomotherapy, proportional to the ratio of
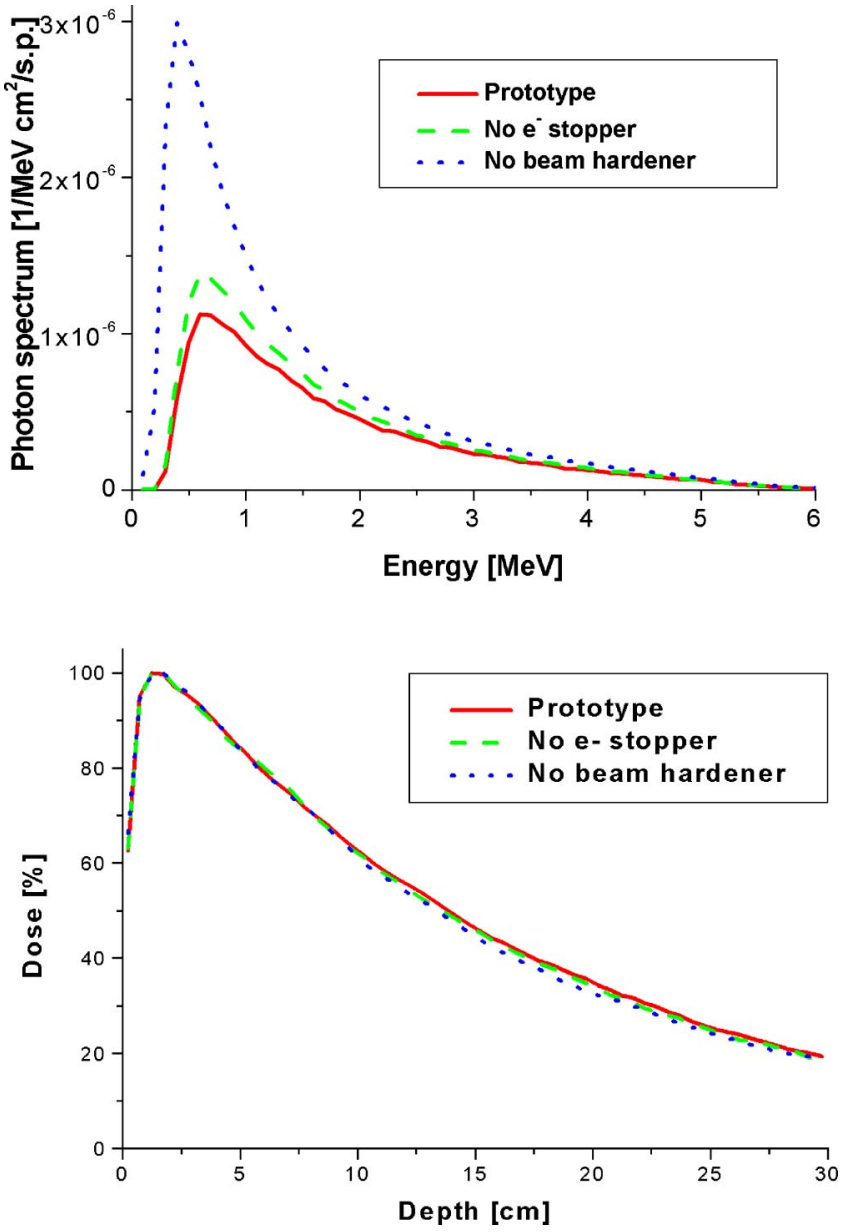

FIG. 10. Sensitivity study of the treatment beam characteristics on different beam modifiers. While the changes in the output photon spectrum are significant (top), the dosimetric characteristics remain almost the same (bottom).

standard and tomotherapy field sizes. Therefore, any gain in the output fluence rate is important in shortening the treatment time.

As seen earlier, beside the primary target, there are other structures in the beam, which we call the extended target. The most important structures are the aluminum electron stopper and tungsten beam hardener. The study of the effect of each of these beam modifiers to the photon beam spectra and consequent dosimetric characteristics are shown in Fig. 10.

It is clearly seen from Fig. 10 that the presence of different beam modifiers only slightly affects percent depth dose distributions, while it significantly affects the output fluence rate. For $6 \mathrm{MeV}$ incident electron energy, where the study was performed, the increase in the fluence rate is about $15 \%$ if there is no electron stopper present and almost 50\% if the beam hardener is taken from the beam. The reason for the percent depth dose insensitivity is that dose distributions, especially at larger depths (past $D_{\max }$ ), are mostly determined by the high energy component of the spectrum, which remains rather unchanged in the presence of different beam modifiers. It should be mentioned that the hardening filter also mitigates the unlikely hazard of a breach in the target 


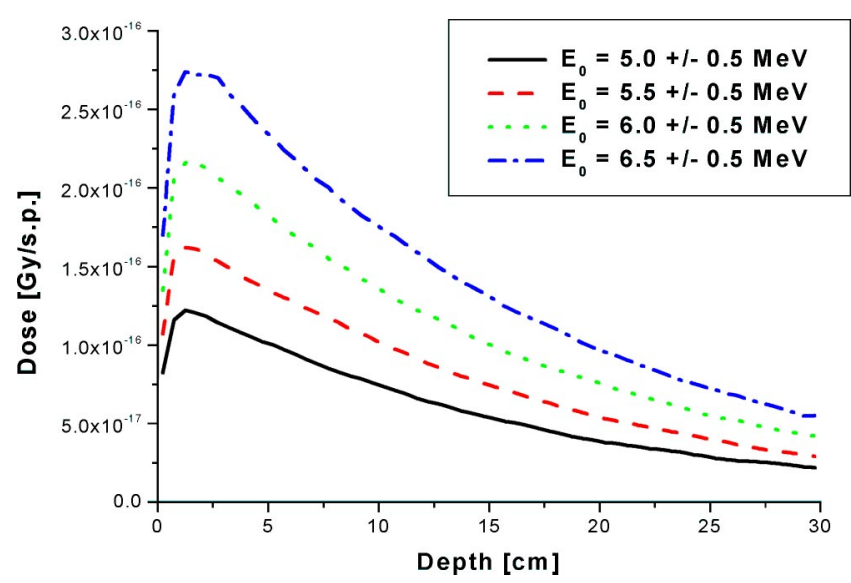

FIG. 11. Sensitivity study of dose output on incident electron energy. A realistic range of the helical tomotherapy energies is considered.

housing by the electron beam (for more discussion see Sec. III F).

Another possibility for an increase of the output fluence rate is to increase the incident electron energy. An increase of the output fluence with the incident electron energy is quite strong, because of the strong dependence of the bremsstrahlung production on the energy. It can be shown that the dose rate output is proportional to $D \propto E_{0}^{3} \ln \left(2 E_{0} / m_{e} c^{2}\right)$, where $E_{0}$ is the incident electron energy and $m_{e} c^{2}$ is the electron mass energy. The integral dose rate gain with the increased energy, compared to the $5 \mathrm{MeV}$ incident electron beam (Fig. 11) is 1.36, 1.84 and 2.38, for 5.5, 6 and $6.5 \mathrm{MeV}$, respectively, which matches well the theoretical prediction of $1.37,1.83$ and 2.39 for $5.5,6$ and $6.5 \mathrm{MeV}$, respectively.

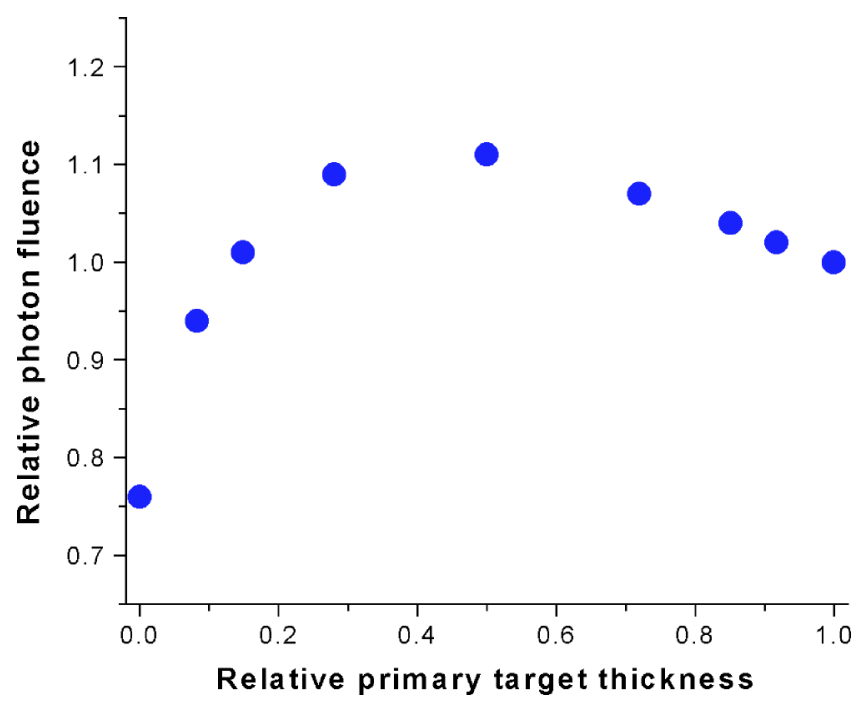

FIG. 12. Relative photon fluence as a function of the primary target thickness compared to the full target photon fluence. Notice the first fluence increase for reduced thicknesses, because of the decreased attenuation and the later fluence decrease because of the lower electron-to-photon conversion of the extended target compared to the primary target.
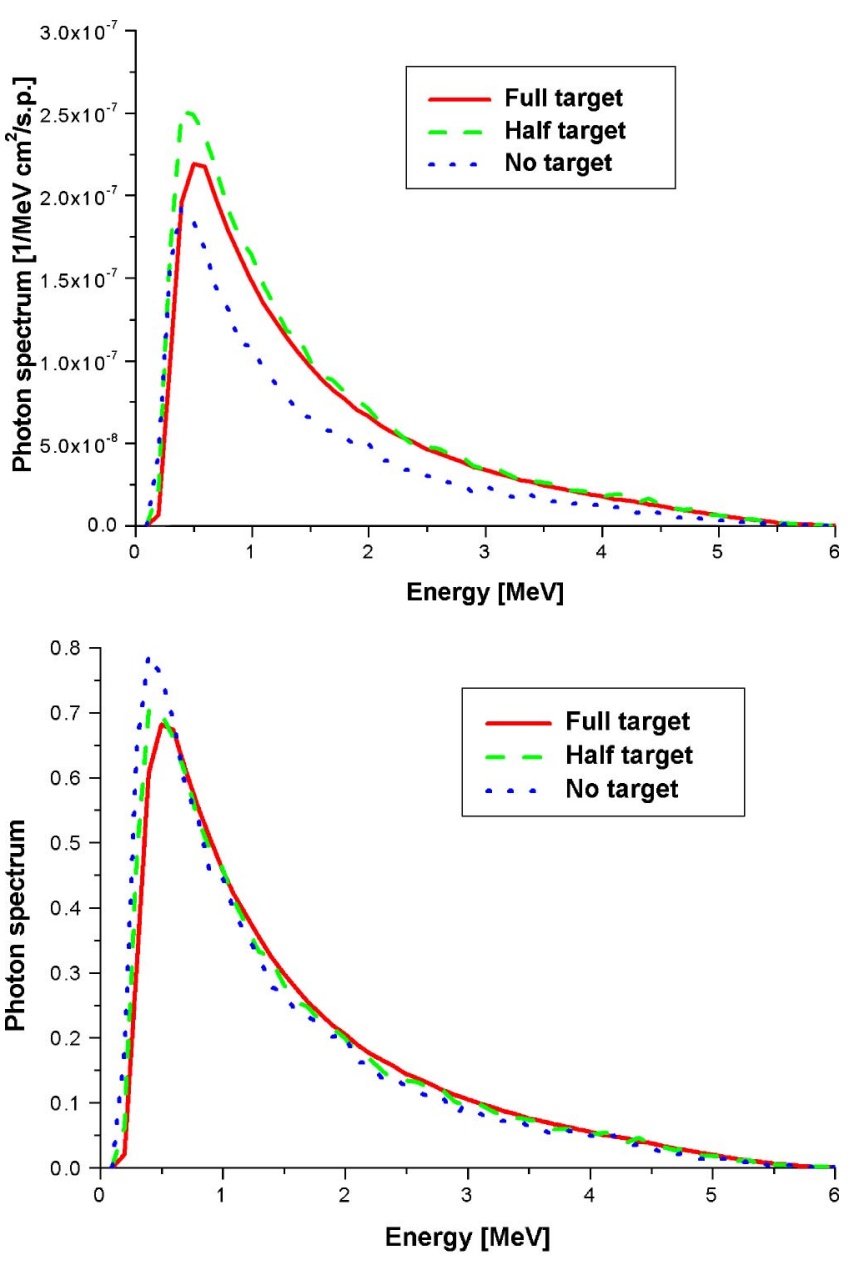

FIG. 13. Absolute (top) and normalized (bottom) photon spectra for various target thicknesses. Note the absolute photon fluence increase for half target thickness and decrease for no primary target, with little relative change in the spectra.

\section{F. Radiation safety}

Not having the flattening filter in the radiation beam potentially raises safety concerns in the event that the primary target were to deteriorate (or be absent in the extreme case) and the electron beam could directly hit a patient. In all other treatment units, the flattening filter plays the role of an "emergency beam stopper," if the target would be damaged. A study of the target thickness on the output photon fluence was conducted and the results are shown in Fig. 12.

Initially when the target thickness decreases the photon fluence increases. The main reason for the increase is the decreased attenuation of the photon beam. If the target thickness decreases further, or if no target is present, the photon fluence drops, however only to approximately $75 \%$ of the full target output. This fluence drop is caused by the decreased electron-to-photon conversion. It is interesting to note that the photon fluence does not drop to zero as one might expect. The reason for the relatively high photon fluence is the rest of the material in the beam, which should be seen as a part of an extended target. Since no dramatic changes in the photon fluence are expected (as shown earlier, a similar magnitude of the output change could be obtained 


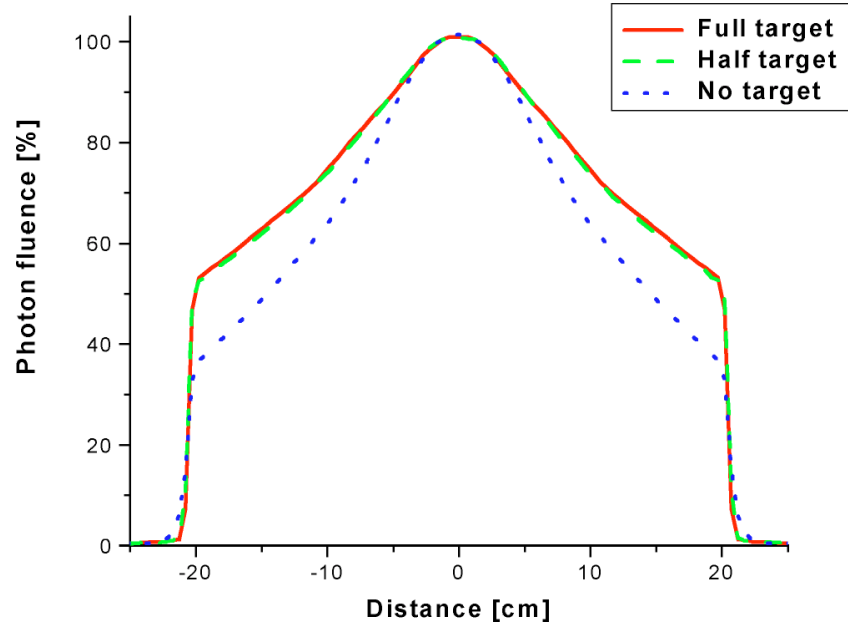

FIG. 14. Relative photon fluence profiles for various target thicknesses. Notice a more forward-peaked photon beam because of the decreased electron scattering on the extended target, because of the lower atomic number compared to the primary target.

from changing the initial electron energy by only approximately $5 \%$ ), the question arises as to whether one could detect a potential problem with the target at all. For this reason other radiation characteristics were examined. The photon fluence spectrum and the transverse profiles are given in Figs. 13 and 14. It is interesting to note that while the spectrum change is rather small (the average energy drops for only $3.5 \%$ and $10 \%$ for half and no target, respectively), the change in the transverse profile cone angle is much more significant, since the ratio of the $20 \mathrm{~cm}$ off-axis to the central-axis profile drops from approximately $50 \%$ to approximately $35 \%$. The reason is the lower average atomic number of the extended target compared to the primary target and consequent smaller average electron scattering angle.

\section{CONCLUSIONS}

Since the helical tomotherapy unit is a dedicated IMRT device with on-board MVCT imaging capability, its radiation beam characteristics are in several aspects different from characteristics of other treatment units. In our study, simulations of the helical tomotherapy prototype installed at UW Hospitals in Madison, WI (Hi-ART I) are presented, however, its radiation characteristics are almost identical to commercial treatment units (Hi-ART II).

Helical tomotherapy has two modes of operation: a treatment mode with approximately $6 \mathrm{MV}$ beam and an MVCT imaging mode with approximately 3.5 MV beam. The photon fluence spectra in the treatment mode were found to be comparable to other radiotherapy treatment systems. Because there is no flattening filter, the change of the off-axis spectrum is very minor. There is less than $5 \%$ change in the mean energy across the fan beam (approximately $40 \times 5 \mathrm{~cm}$ open field size), which is significantly lower than in any other treatment unit. This characteristic is also very favorable for the treatment planning system, since no corrections are needed to account for beam hardening/softening. A lack of the flattening filter also causes the beam intensity to be ap- proximately doubled in the center compared to the edge of an open field and has the characteristic conical shape. Because of the increased collimator shielding, the leakage radiation was calculated to be below $0.05 \%$ and therefore, the in-field scatter dominates the radiation field outside the treatment area. Two possibilities for increasing the output fluence rate were considered; one was the change in the extended target configuration and second was the increase of the incident electron energy. While the change in the extended target configuration can increase the output fluence, the increased energy results in a significantly higher output fluence increase. The radiation safety concern because of the absent flattening filter in case of the damaged target was addressed by establishing only a moderate dose output decrease by approximately $15 \%$ even for the cases where the primary target would be completely absent.

From the presented Monte Carlo studies it can be seen that helical tomotherapy is a system with some unique radiation characteristics, which have been to a large extent optimized for intensity modulated delivery. Increased output fluence in the center of the field, low spectral variation across the field and extremely low leakage radiation distinguish the system from other conventional treatment systems.

\section{ACKNOWLEDGMENTS}

The authors would like to thank Dan Wenman from Physical Sciences Laboratory of University of WisconsinMadison for providing insightful information about the original helical tomotherapy design. This work was supported by the National Cancer Institute grant (P01 CA088960) and TomoTherapy Inc., Madison, WI.

a) Author to whom correspondence should be addressed: Robert Jeraj, Department of Medical Physics, University of Wisconsin-Madison, 1530 MSC, 1300 University Ave., Madison, Wisconsin 53706. Telephone: (608) 263 8619; fax: (608) 262 2413; electronic mail: rjeraj@wisc.edu

${ }^{1}$ J. A. Antolak, M. R. Bieda, and K. R. Hogstrom, "Using Monte Carlo methods to commission electron beams: a feasibility study," Med. Phys. 29, 771-786 (2002).

${ }^{2}$ M. R. Bieda, J. A. Antolak, and K. R. Hogstrom, "The effect of scattering foil parameters on electron-beam Monte Carlo calculations," Med. Phys. 28, 2527-2534 (2001).

${ }^{3}$ E. L. Chaney, T. J. Cullip, and T. A. Gabriel, "A Monte Carlo study of accelerator head scatter,” Med. Phys. 21, 1383-1390 (1994).

${ }^{4}$ F. Z. Company and B. J. Allen, "Measurements and Monte Carlo simulations of the fluence and dose characteristics of microplanar photon beams," Australas. Phys. Eng. Sci. Med. 19, 217-224 (1996).

${ }^{5}$ J. Deng, S. B. Jiang, A. Kapur, J. Li, T. Pawlicki, and C. M. Ma, "Photon beam characterization and modelling for Monte Carlo treatment planning,"' Phys. Med. Biol. 45, 411-427 (2000).

${ }^{6}$ G. X. Ding, "Energy spectra, angular spread, fluence profiles and dose distributions of 6 and $18 \mathrm{MV}$ photon beams: results of Monte Carlo simulations for a Varian 2100EX accelerator," Phys. Med. Biol. 47, 1025-1046 (2002).

${ }^{7}$ M. A. Ebert, P. W. Hoban, and P. J. Keall, "Modelling clinical accelerator beams: a review," Australas. Phys. Eng. Sci. Med. 19, 131-150 (1996).

${ }^{8}$ B. A. Faddegon, P. O'Brien, and D. L. Mason, "The flatness of Siemens linear accelerator X-ray fields," Med. Phys. 26, 220-228 (1999).

${ }^{9}$ K. Han, D. Ballon, C. Chui, and R. Mohan, "Monte Carlo simulation of a cobalt-60 beam," Med. Phys. 14, 414-419 (1987).

${ }^{10}$ N. R. Harper, P. E. Metcalfe, P. W. Hoban, and W. H. Round, "Electron contamination in $4 \mathrm{MV}$ and $10 \mathrm{MV}$ radiotherapy x-ray beams," Australas. Phys. Eng. Sci. Med. 14, 141-145 (1991). 
${ }^{11}$ C. L. Hartmann Siantar, R. S. Walling, T. P. Daly, B. Faddegon, N. Albright, P. Bergstrom, A. F. Bielajew, C. Chuang, D. Garrett, R. K. House, D. Knapp, D. J. Wieczorek, and L. J. Verhey, "Description and dosimetric verification of the PEREGRINE Monte Carlo dose calculation system for photon beams incident on a water phantom," Med. Phys. 28, 1322-1337 (2001).

${ }^{12}$ S. B. Jiang, A. Kapur, and C. M. Ma, "Electron beam modeling and commissioning for Monte Carlo treatment planning," Med. Phys. 27, 180-191 (2000).

${ }^{13}$ A. Kapur, C. M. Ma, E. C. Mok, D. O. Findley, and A. L. Boyer, "Monte Carlo calculations of electron beam output factors for a medical linear accelerator," Phys. Med. Biol. 43, 3479-3494 (1998).

${ }^{14}$ P. J. Keall and P. W. Hoban, "The angular and energy distribution of the primary electron beam," Australas. Phys. Eng. Sci. Med. 17, 116-123 (1994).

${ }^{15}$ P. C. Lee, "Monte Carlo simulations of the differential beam hardening effect of a flattening filter on a therapeutic x-ray beam," Med. Phys. 24, 1485-1489 (1997).

${ }^{16}$ R. D. Lewis, S. J. Ryde, D. A. Hancock, and C. J. Evans, "An MCNPbased model of a linear accelerator x-ray beam," Phys. Med. Biol. 44, 1219-1230 (1999).

${ }^{17}$ S. Y. Lin, T. C. Chu, and J. P. Lin, "Monte Carlo simulation of a clinical linear accelerator," Appl. Radiat. Isot. 55, 759-765 (2001).

${ }^{18}$ C. M. Ma and S. B. Jiang, "Monte Carlo modelling of electron beams from medical accelerators," Phys. Med. Biol. 44, R157-189 (1999).

${ }^{19}$ R. Mohan, C. Chui, and L. Lidofsky, "Energy and angular distributions of photons from medical linear accelerators," Med. Phys. 12, 592-597 (1985).

${ }^{20}$ G. M. Mora, A. Maio, and D. W. Rogers, "Monte Carlo simulation of a typical 60Co therapy source," Med. Phys. 26, 2494-2502 (1999).

${ }^{21}$ P. L. Petti, M. S. Goodman, J. M. Sisterson, P. J. Biggs, T. A. Gabriel, and R. Mohan, "Sources of electron contamination for the Clinac-35 25-MV photon beam," Med. Phys. 10, 856-861 (1983).

${ }^{22} \mathrm{D}$. W. Rogers, "The role of Monte Carlo simulation of electron transport in radiation dosimetry," Int. J. Rad Appl. Instrum. [A] 42, 965-974 (1991).

${ }^{23}$ A. E. Schach von Wittenau, L. J. Cox, P. M. Bergstrom, Jr., W. P. Chandler, C. L. Hartmann Siantar, and R. Mohan, "Correlated histogram representation of Monte Carlo derived medical accelerator photon-output phase space," Med. Phys. 26, 1196-1211 (1999).

${ }^{24}$ J. Sempau, A. Sanchez-Reyes, F. Salvat, H. O. ben Tahar, S. B. Jiang, and J. M. Fernandez-Varea, "Monte Carlo simulation of electron beams from an accelerator head using PENELOPE," Phys. Med. Biol. 46, 1163-1186 (2001).

${ }^{25}$ D. Sheikh-Bagheri and D. W. Rogers, "Monte Carlo calculation of nine megavoltage photon beam spectra using the BEAM code," Med. Phys. 29, 391-402 (2002).

${ }^{26}$ J. V. Siebers, P. J. Keall, B. Libby, and R. Mohan, "Comparison of EGS4 and MCNP4b Monte Carlo codes for generation of photon phase space distributions for a Varian 2100C," Phys. Med. Biol. 44, 3009-3026 (1999).

${ }^{27} \mathrm{~W}$. van der Zee and J. Welleweerd, "Calculating photon beam characteristics with Monte Carlo techniques," Med. Phys. 26, 1883-1892 (1999).

${ }^{28}$ F. Verhaegen, C. Mubata, J. Pettingell, A. M. Bidmead, I. Rosenberg, D. Mockridge, and A. E. Nahum, "Monte Carlo calculation of output factors for circular, rectangular, and square fields of electron accelerators (6-20 MeV)," Med. Phys. 28, 938-949 (2001).

${ }^{29}$ F. Verhaegen and J. P. Seuntjens, "Monte Carlo modelling of external radiotherapy photon beams," Phys. Med. Biol. 48, R107-R163 (2003).

${ }^{30}$ E. Wieslander and T. Knoos, "A virtual linear accelerator for verification of treatment planning systems," Phys. Med. Biol. 45, 2887-2896 (2000).

${ }^{31}$ D. W. Rogers, B. A. Faddegon, G. X. Ding, C. M. Ma, J. We, and T. R. Mackie, "BEAM: a Monte Carlo code to simulate radiotherapy treatment units," Med. Phys. 22, 503-524 (1995).

${ }^{32}$ W. R. Nelson, H. Hirayama, and D. W. Rogers, "The EGS4 code system," Report No. 265, Stanford Linear Accelerator Center, Stanford, CA, 1985.

${ }^{33}$ I. Kawrakow and D. W. Rogers, "The EGsnrc Code System: Monte Carlo simulation of electron and photon transport," Report No. PIRS-701, National Research Council of Canada, Ottawa, Canada, 2000.

${ }^{34}$ J. Briesmeister, "MCNP-A general Monte Carlo $N$-particle transport code, Verison 4A," Report No. LA-12625, Los Alamos National Laboratory, Los Alamos, NM, 1999.

${ }^{35}$ J. Baro, J. Sempau, J. M. Fernandez-Varea, and F. Salvat, "PENELoPE: An algorithm for Monte Carlo simulation of the penetration and energy loss of electrons and positrons in matter," Nucl. Instrum. Methods Phys. Res. B 100, 31-46 (1995).

${ }^{36}$ T. R. Mackie, T. Holmes, S. Swerdloff, P. Reckwerdt, J. O. Deasy, J. Yang, B. Paliwal, and T. Kinsella, "Tomotherapy: a new concept for the delivery of dynamic conformal radiotherapy," Med. Phys. 20, 1709-1719 (1993).

${ }^{37}$ E. J. Hall and C. S. Wuu, "Radiation-induced second cancers: the impact of 3D-CRT and IMRT," Int. J. Radiat. Oncol., Biol., Phys. 56, 83-88 (2003). 